\section{Chuzan Virus in Yaks, Qinghai-Tibetan Plateau, China}

\author{
Meng Wang, Yun Wang, Abdul Rasheed Baloch, \\ Yangyang Pan, Lili Tian, Fang $\mathrm{Xu}$, \\ Shaobo Chen, Qiaoying Zeng
}

Author affiliations: Gansu Agricultural University, Lanzhou, China (M. Wang, Y. Pan, F. Xu, Q. Zeng); Anning Branch Lanzhou General Hospital, Lanzhou (Y. Wang); University of South Bohemia in České Budějovice, Zátiší Vodňany, Czech Republic (A.R. Baloch); China Animal Health and Epidemiology Center, Qingdao, China (L. Tian); Veterinary Department of Gansu Province, Lanzhou (S. Chen)

DOI: https://doi.org/10.3201/eid2412.171414

We detected Chuzan virus (CHUV) in domestic yaks from the Qinghai-Tibetan Plateau, western China, indicating CHUV probably has been transmitted to yaks in recent years. Awareness for CHUV surveillance and transmission and livestock health management in these special regions should be raised to avoid outbreaks and animal loss.

$\mathrm{C}$ huzan virus (CHUV) belongs to the Palyam serogroup of genus Orbivirus, family Reoviridae. A CHUV outbreak, first reported in Japan in 1985, was the causative agent of disease that resulted in many reproductive disorders in cattle, including abortion, stillbirth, and congenital malformation ( 1 ) and in considerable economic loss in the cattle industry.

Like other orbiviruses, CHUV consists of 10 doublestranded RNA segments (Seg-1 to Seg-10), which encode 7 structural viral proteins (VP1-VP7) and 4 nonstructural proteins (NS1-NS4) (2-4). Seg-2 (VP2) and Seg-6 (VP5) of the Palyam serogroup show the highest levels of variation in genome sequence, which correlates with virus serotype specificity (2). These viruses usually are transmitted by arthropod vectors $(5,6)$. Therefore, CHUV has been widespread in many countries of Asia, such as South Korea (7) and mainland China $(8,9)$, which have reported CHUV infection in cattle. However, no information was available about CHUV in yaks (Bos grunniens) on China's QinghaiTibetan Plateau.

Yaks are an important livestock in the Qinghai-Tibetan Plateau. They have been farmed with other livestock, such as Tibetan sheep and Tibetan pigs. The high prevalence of bluetongue virus (BTV) infection, also belonging to genus Orbivirus, has been reported in yaks and Tibetan sheep (10). A study in 2016 found an abortion rate in yaks of $21.39 \%$ in part of Qinghai Province, presumably because of the high prevalence of BTV and other related pathogens (10). All these data and CHUV infection in cattle in China motivated us to study whether CHUV infects yaks.

During August 2016-April 2017, we randomly collected 208 blood samples from apparently healthy domestic yaks, 71 yaks from Gansu Province $(46<1$ year of age), 64 yaks from Qinghai Province $(23<1$ year of age), and 73 yaks from Sichuan Province $(29<1$ year of age). Soon after sampling, total RNAs were extracted and used as templates to amplify full-length cDNA by reverse transcription PCR (RT-PCR; SuperScript III Synthesis Kit, Invitrogen, Carlsbad, CA, USA). One pair of specific primers was designed based on VP2 genome sequence of CHUV (online Technical Appendix Table 1, https://wwwnc.cdc. gov/EID/article/24/12/17-1414-Techapp1.pdf) and used to detect CHUV in yaks. We also performed serologic assay by using the CHUV 2nd detection kit (iNtRON, IPC11028, Gyeonggi-do, South Korea), and the results of the assay were then authenticated by RT-PCR.

For phylogenetic and identity analysis of genome sequence of 10 segments from CHUV, we designed 10 pairs of primer based on known sequences deposited in GenBank (online Technical Appendix Table 1) to obtain the open reading frame genome of these proteins. Phylogenetic and identity analyses were performed based on these genome sequences and the corresponding sequences available in GenBank.

Five (7\%) of the 71 samples were positive for CHUV in Gansu Province, 4 of which were $<1$ year of age (Figure; online Technical Appendix Table 2). CHUV infection in yaks was not found in Qinghai and Sichuan provinces. Relatively low prevalence of CHUV infection in yaks is consistent with the report of CHUV infection in cattle in South Korea (7), which was also significantly lower than BTV infection in yaks (17.34\%) (10). We also obtained similar results in serologic investigation. We observed neither illness nor disease in these yaks; our results are consistent with CHUV infection in sentinel cattle (8) and thus suggest that subclinical infection of CHUV occurs in cattle. We used heparinized blood samples to inoculate baby hamster kidney 21 cells for 5 blind passages, as described previously $(6,8,9)$. Infected cells that exhibited a wrinkled morphology and were detached from the bottom of culture flasks within 5 days (online Technical Appendix Figure 1), and 19 segments of CHUV could also be detected by RT-PCR.

Sequence analysis revealed $100 \%$ identity of genomes for Seg-1 to Seg-10 of 3 CHUV sequences in yaks (CHN-GS-70). Identity analysis showed that genome sequences for Seg-1 to Seg-10 of CHUV shared $>98.38 \%$ nt identities and $>98.09 \%$ aa identities with CHUV strain KT887181/GX871/China in previous studies $(8,9)$ (online Technical Appendix Table 3). We constructed 2 phylogenetic trees based on VP2 and VP5 genome sequences 


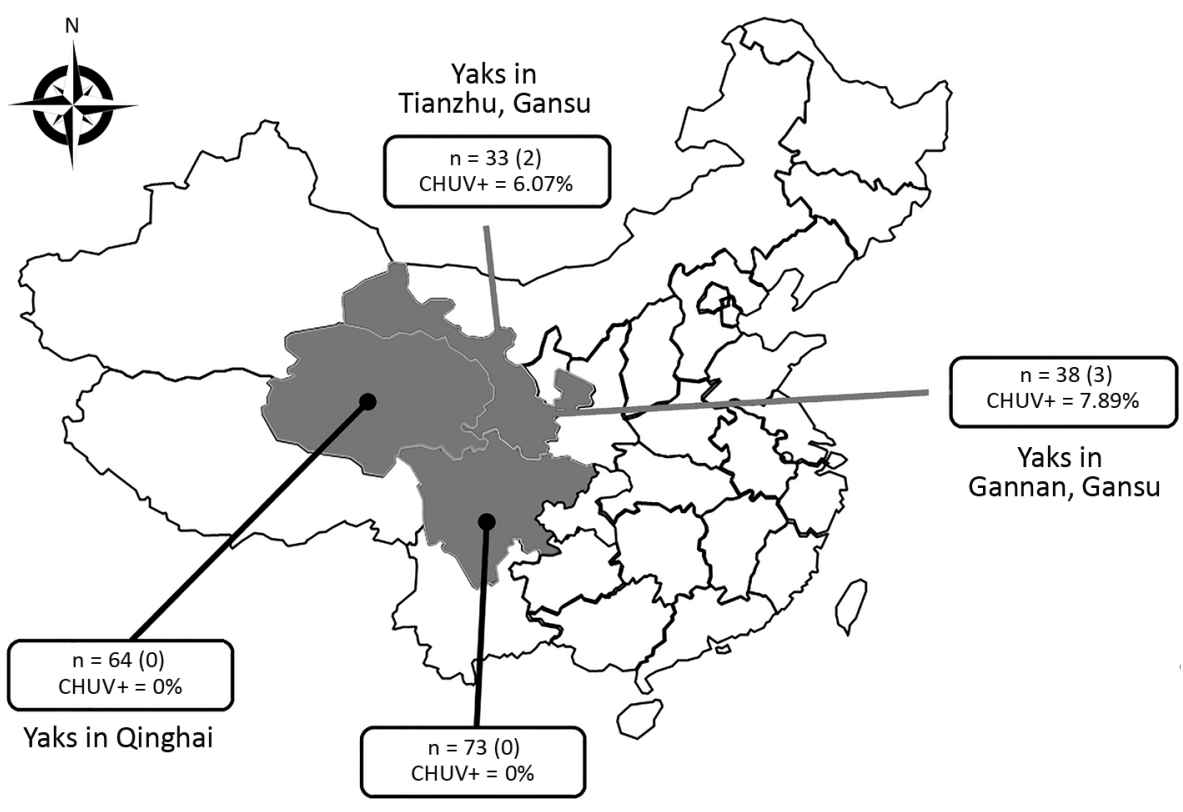

Figure. Number and species of yaks from provinces around the Qinghai Tibetan Plateau, China, 2016-2017. The 3 provinces where sampling was performed, yak species, and occurrence of CHUV are indicated. $n$ values indicate the total number of samples in each province; numbers in parentheses indicate the numbers of positive samples in each province; $\mathrm{CHUV}+$ percentages indicate the CHUV prevalence rate. CHUV, Chuzan virus.

Yaks in Sichuan

of CHUV and other members in genus Orbivirus (online Technical Appendix Figures 2, 3). All strains from our study were grouped in a new separate cluster and shared an ancestor with the strains KT002589/SZ187/China and KT887181/GX871/China. Furthermore, CHN-GS-26 and CHN-GS-70 were located in the same separate sub-cluster (online Technical Appendix Figures 2, 3), which demonstrated a complicated and transregional transmission cycle for CHUV in China.

The yaks that were positive for CHUV were located in 2 cities of Gansu Province $\approx 600 \mathrm{~km}$ apart, which indicates that transmission of CHUV has spread rapidly around the Qinghai-Tibetan Plateau. Further studies are needed to determine the epidemiology and evolution of CHUV in livestock with concomitant virus isolation and phylogenetic analysis. The awareness of livestock health management in these special regions should also be raised.

\section{Acknowledgment}

We thank the Veterinary Department of Gansu Province for its valuable assistance in sample collection.

This work was supported by a grant from the National Natural Science Foundation of China no. 31260616; the Fuxi Foundation of Exceptional Talent at Gansu Agricultural University and the grant from Gansu Agricultural University, College of Veterinary Medicine; the Scientific Research Foundation for New Scholars, Gansu Agricultural University (no. GSAURCZX201702); and the Innovation Foundation of the College of Veterinary Medicine, Gansu Agriculture University (no. JYCX-KX017).

\section{About the Author}

Dr. Wang is a veterinary pharmacologist and epidemiologist at the College of Veterinary Medicine from Gansu Agricultural University. Her primary research interests are veterinary pharmacology, animal disease impact in the Qinghai-Tibetan Plateau of China, and the role of livestock in infectious disease epidemiology.

\section{References}

1. Miura Y, Goto Y, Kubo M, Kono Y. Isolation of Chuzan virus, a new member of the Palyam subgroup of the genus Orbivirus, from cattle and Culicoides oxystoma in Japan. Am J Vet Res. 1988;49:2022-5.

2. Ohashi S, Matsumori Y, Yanase T, Yamakawa M, Kato T, Tsuda T. Evidence of an antigenic shift among Palyam serogroup orbiviruses. J Clin Microbiol. 2004;42:4610-4. http://dx.doi.org/ 10.1128/JCM.42.10.4610-4614.2004

3. Belhouchet M, Mohd Jaafar F, Firth AE, Grimes JM, Mertens PP, Attoui H. Detection of a fourth orbivirus non-structural protein. PLoS One. 2011;6:e25697. http://dx.doi.org/10.1371/ journal.pone. 0025697

4. Ratinier M, Caporale M, Golder M, Franzoni G, Allan K, Nunes SF, et al. Identification and characterization of a novel non-structural protein of bluetongue virus. PLoS Pathog. 2011;7:e1002477. http://dx.doi.org/10.1371/journal.ppat.1002477

5. Rao PP, Hegde NR, Reddy YN, Krishnajyothi Y, Reddy YV, Susmitha B, et al. Epidemiology of bluetongue in India. Transbound Emerg Dis. 2016;63:e151-64. http://dx.doi. org/10.1111/tbed.12258

6. Wang J, Li H, He Y, Zhou Y, Xin A, Liao D, et al. Isolation of Tibet orbivirus from Culicoides and associated infections in livestock in Yunnan, China. Virol J. 2017;14:105. http://dx.doi.org/10.1186/ s12985-017-0774-9

7. Lim SI, Kweon CH, Tark DS, Kim SH, Yang DK. Sero-survey on Aino, Akabane, Chuzan, bovine ephemeral fever and Japanese encephalitis virus of cattle and swine in Korea. J Vet Sci. 2007; 8:45-9. http://dx.doi.org/10.4142/jvs.2007.8.1.45 
8. Wang F, Lin J, Chang J, Cao Y, Qin S, Wu J, et al. Isolation, complete genome sequencing, and phylogenetic analysis of the first Chuzan virus in China. Virus Genes. 2016;52:138-41. http://dx.doi.org/10.1007/s11262-015-1282-x

9. Yang H, Xiao L, Meng J, Xiong H, Gao L, Liao D, et al. Complete genome sequence of a Chuzan virus strain isolated for the first time in mainland China. Arch Virol. 2016;161:1073-7. http://dx.doi.org/10.1007/s00705-015-2734-2

10. Ma J-G, Zhang X-X, Zheng W-B, Xu Y-T, Zhu X-Q, Hu G-X, et al. Seroprevalence and risk factors of bluetongue virus infection in Tibetan sheep and yaks in Tibetan Plateau, China. Biomed Res Int. 2017;2017:5139703.

Address for correspondence: Qiaoying Zeng, Gansu Agricultural University, The College of Veterinary Medicine, Lanzhou, China; email: zengqy@gsau.edu.cn

\section{Conservation of White Rhinoceroses Threatened by Bovine Tuberculosis, South Africa, 2016-2017}

\section{Michele A. Miller, ${ }^{1}$ Peter Buss, ${ }^{1}$ \\ Sven D.C. Parsons, Eduard Roos, Josephine Chileshe, Wynand J. Goosen, Louis van Schalkwyk, Lin-Mari de Klerk-Lorist, Markus Hofmeyr, ${ }^{2}$ Guy Hausler, Leana Rossouw, Tebogo Manamela, Emily P. Mitchell, ${ }^{3}$ Rob Warren, Paul van Helden}

\begin{abstract}
Author affiliations: Stellenbosch University, Department of Science and Technology-National Research Foundation Centre of Excellence for Biomedical Tuberculosis Research, South African Medical Research Council Centre for Tuberculosis Research, Cape Town, South Africa (M.A. Miller, S.D.C. Parsons, E. Roos, J. Chileshe, W.J. Goosen, G. Hausler, R. Warren, P. van Helden); South African National Parks, Skukuza, South Africa (P. Buss, M. Hofmeyr, L. Rossouw, T. Manamela); Office of the State Veterinarian, Skukuza (L. van Schalkwyk, L.-M. de Klerk-Lorist); National Zoological Gardens of South Africa, Pretoria, South Africa (E.P. Mitchell)
\end{abstract}

DOI: https://doi.org/10.3201/eid2412.180293

\footnotetext{
${ }^{1}$ These first authors contributed equally to this article.

${ }^{2}$ Current affiliation: Great Plains Conservation, Maun, Botswana.

${ }^{3}$ Current affiliation: University of Pretoria, Pretoria, South Africa.
}

During 2016-2017, when Kruger National Park, South Africa, was under quarantine to limit bovine tuberculosis spread, we examined 35 white and 5 black rhinoceroses for infection. We found 6 infected white rhinoceroses during times of nutritional stress. Further research on Mycobacterium bovis pathogenesis in white rhinoceroses is needed.

$\mathrm{T}$ uberculosis (TB) caused by Mycobacterium tuberculosis or M. bovis has been reported in captive rhinoceroses since the early 1800 s (1-3). Bovine TB is endemic in many wildlife populations worldwide, including among those in Kruger National Park (KNP), South Africa (4). KNP contains the largest free-living population of white rhinoceroses in the world (estimated at 6,6497,830). However, prolonged drought in South Africa (2015-2017) raised concerns that starvation and disease could increase the mortality rate and affect conservation efforts for this species (5).

In June 2016, a black rhinoceros (Diceros bicornis minor) with an M. bovis infection was discovered (6). Thereafter, a surveillance program was initiated to screen rhinoceros carcasses in KNP, leading to 35 white and 5 black rhinoceros carcasses being examined during June 2016-October 2017. To determine which animals were infected, we conducted macroscopic examinations and collected samples for histopathologic studies and mycobacterial culture, as previously described (7). Research protocols were approved by the South African National Park Animal Use and Care Committee and ethics committee of Stellenbosch University.

No additional cases of $M$. bovis infection were found in black rhinoceroses. However, we confirmed M. bovis infection in 6 white rhinoceroses (Table). Grossly visible lesions, mostly found in the retropharyngeal or tracheobronchial lymph nodes or lung, were typically small and localized and could easily be missed or mistaken for granulomas caused by other pathogens if careful dissections of tissues were not performed (online Technical Appendix, https:// wwwnc.cdc.gov/EID/article/24/12/18-0293-Techapp1. pdf). On histologic examination, we found granulomatous inflammation in lung or lymph node sections and rare acidfast organisms in some granulomas (Table). We typed these $M$. bovis isolates as strain SB0121, the most common strain found in KNP (8).

Four of the infected animals were found during September-November 2016, near the end of the drought, and the remaining 2 animals were found in September and October 2017, at the end of the next winter. The timing of infections suggests that animals under nutritional stress might be more susceptible to infection, similar to observations in other species (9). The low number of positive cases and localized paucibacillary lesions support the hypothesis that white rhinoceroses, although susceptible to infection, 